\title{
K-Ras Mutations in Non-Small-Cell Lung Cancer: Prognostic and Predictive Value
}

\author{
Manolo D'Arcangelo and Federico Cappuzzo \\ Istituto Toscano Tumori, Ospedale Civile di Livorno, 57100 Livorno, Italy \\ Correspondence should be addressed to Federico Cappuzzo, f.cappuzzo@googlemail.com
}

Received 10 January 2012; Accepted 6 February 2012

Academic Editor: J. S. Castresana

Copyright ( $) 2012$ M. D’Arcangelo and F. Cappuzzo. This is an open access article distributed under the Creative Commons Attribution License, which permits unrestricted use, distribution, and reproduction in any medium, provided the original work is properly cited.

\begin{abstract}
Non-small-cell lung cancer (NSCLC) is a heterogeneous disease due to the presence of different clinically relevant molecular subtypes. Until today, several biological events have been identified in lung adenocarcinoma, including epidermal growth factor receptor $(E G F R)$ mutations and anaplastic lymphoma kinase $(A L K)$ translocations, offering new hopes to patients with metastatic disease. Unfortunately, in approximately $50 \%$ of adenocarcinoma and for those harbouring $K$ - $R A S$ mutations, the most frequent mutation in Caucasian lung adenocarcinoma, so far no specific drug demonstrated efficacy. The rat sarcoma (RAS) genes, including $H$-RAS, $K-R A S$, and $N$-RAS, encode a family of proteins regulating cell growth, differentiation, and apoptosis. $K-R A S$ mutations are present in 20-30\% of NSCLC and occur most commonly, but not exclusively, in adenocarcinoma histology and life-long smokers. Although in colorectal cancer patients $K-R A S$ mutations represent a validated negative predictive biomarker for treatment with anti-EGFR monoclonal antibodies, their role in selecting specific treatment for NSCLC patients remains undefined. Aim of the present paper is to critically analyze the prognostic and predictive value of $K-R A S$ mutations in NSCLC.
\end{abstract}

\section{Introduction}

In 2011 non-small-cell lung cancer (NSCLC) remains the principal cause of cancer-related death worldwide, accounting for more than one million deaths per year [1]. Therapeutic progresses have signed out the last decade, but median survival for patients in advanced stage is still disappointing [2]. NSCLC accounts for $80 \%$ of lung tumors, including adenocarcinoma in $35-40 \%$ of cases, squamous cell carcinoma in 25-30\%, and large cell carcinoma in 10$15 \%$. For many years we treated metastatic NSCLC with the same regimens, irrespective of any clinical or biological characteristics. Today, histology seems a relevant parameter for defining the best regimen, with new agents, such as pemetrexed and bevacizumab, effective and safe only in nonsquamous populations [3, 4]. During the last few years, improvement in the knowledge of lung cancer biology led to identification of molecular events crucial for tumor cell survival. Cancer cell survival might depend on the expression of a single-mutant oncogene according to a model called "oncogene addiction" $[5,6]$. In NSCLC a number of driving mutations have been identified, including Epidermal growth factor receptor (EGFR) mutations, KRAS mutations, HER2 mutations and EML4-ALK translocations. Since their identification in 2004, activating EGFR gene mutations have emerged as the most relevant predictor of response to a class of compounds, the EGFR tyrosine kinase inhibitors (EGFRTKIs) gefitinib and erlotinib. Six phase III randomized trials demonstrated that patients harboring activating EGFR mutations benefit more from EGFR-TKIs than from standard platinum-based chemotherapy at least in terms of response rate (RR), progression-free survival (PFS), toxicity profile and quality of life [7-12]. Randomized phase III trials in the maintenance setting (SATURN and ATLAS), in secondline versus chemotherapy (INTEREST and TITAN) and versus placebo (BR21) confirmed the high efficacy of EGFRTKIs in the presence of activating EGFR mutations [13-17]. Today in patients harbouring an EGFR mutation, gefitinib or erlotinib represent the best therapeutic option irrespectively of treatment line. Nevertheless, large randomized clinical 
trials demonstrated that erlotinib could produce a modest benefit even in the EGFR wild-type population $[13,14]$.

Therefore, a relevant issue in clinical practice is the identification of EGFR wild-type patients that could benefit or that could be excluded from an EGFR-TKI therapy. Unfortunately, at present, there is no single biomarker that could be used for precluding the treatment to any patient, including $K-R A S$ mutations [14]. Although in colorectal cancer $K$-RAS mutations are the most useful biomarker for selecting patients who are candidate for treatment with antiEGFR monoclonal antibodies, cetuximab or panitumumab, its role in NSCLC as prognostic or predictive marker is less defined [18]. The aim of the present paper is to analyze the role of K-RAS mutations in NSCLC.

\section{RAS Mutations in NSCLC}

The RAS gene family includes $H$-RAS, K-RAS and N-RAS and encodes for membrane-bound $21-\mathrm{kd}$ guanosinetriphosphate-(GTP-) binding proteins regulating cell growth, differentiation and apoptosis by interacting with multiple effectors including mitogen-activated protein kinase (MAPK), phosphoinositide 3-kinase (PI3K) and signal transducer and activator of transcription (STAT) cascades (Figure 1). RAS proteins acquire transforming potential when a point mutation in the gene replaces an amino acid at position 12, 13 or 61 [19]. These mutations lead to forms of RAS with impaired GTPase activity, causing a constitutive activation of RAS signalling pathway. Mutations in K-RAS gene occur frequently in NSCLC [20], more frequently $(20-30 \%)$ in adenocarcinoma and less frequently (about 7\%) in squamous-cell carcinoma [21]. In NSCLC the vast majority of $K-R A S$ mutations involve codons 12 or 13 and are usually associated with a history of tobacco use [22]. K-RAS mutations frequency varies among different ethnic groups, with lower frequency observed among Asians and higher frequency among African Americans compared to white Caucasians [23]. Recently K-RAS mutations have been detected in a significant proportion of never smoker NSCLC patients, with an incidence up to $15 \%$ [23]. Thus, unlike EGFR mutations, which occur more frequently in never smokers, presence of a $K-R A S$ mutation cannot be predicted on the basis of smoking history alone.

\section{K-RAS Mutation as Prognostic Biomarker}

The role of $K-R A S$ mutations as a prognostic factor in NSCLC remains controversial. Although some studies suggested a potential negative prognostic effect, other studies did not confirm any negative impact on survival for individuals harbouring a $K-R A S$ mutation. More than 50 studies have been published, using different methods for K-RAS testing and with conflicting results (Table 1). In an ancillary study of JBR.10 trial, a phase III trial of adjuvant chemotherapy versus observation in resected NSCLC, among the 450 analyzed cases, $26 \%$ harboured a $K$-RAS mutation [24]. In the group of patients not treated with chemotherapy, $K-R A S$ mutations were not prognostic for survival $(P=0.4)$. In the
E4592 trial, another phase III trial of adjuvant chemotherapy versus observation in resected NSCLC, 24\% of 184 assessable tumors were positive for K-RAS mutations [25]. The median survival of mutated and wild-type patients was not statistically different (30 and 42 months, resp. $P=0.38$ ). Graziano et al. investigated the prognostic effect of $K-R A S$ mutations in stage I and II resected NSCLC [26]. In the whole population, no statistical difference was found in OS for $K$ $R A S$-mutations-positive and negative patients $(P=0.33)$. Keohavong et al. found no association of K-RAS mutation and survival in 173 adenocarcinoma and adenosquamous NSCLC patients [27]. In another study, Lu et al. evaluated the prognostic role of a panel of six biomarkers including $K$-RAS mutations, in completely resected stage I NSCLC [28]. Patients were followed up for a minimum of 5 years; $K$-RAS mutations were detected in $34 \%$ of samples and were not associated with overall survival $(P=0.517)$. Conversely, Slebos reported a series of 69 surgically treated adenocarcinomas of the lung in which K-RAS codon-12 point mutations resulted in a negative prognostic factor for disease-free survival $(P=0.038)$ and overall survival $(P=$ 0.002 ) [29]. This difference was consistent also after adjustment for factors such as stage, tumor size and differentiation. In a prospective series of 365 patients with resected early stage NSCLC treated at Massachusetts General Hospital, $K$-RAS mutations were found only in smokers and were associated with worse survival $(P=0.009$, log-rank test $)$ only in stage I disease, but not in the whole population [30]. In a Japanese study, Fukuyama et al. examined 159 cases of NSCLC for mutation at codon 12 of $K$-RAS gene and found $6.9 \%$ of mutated patients [31]. The $K$-RAS mutation positive group had a worse survival than the $K$-RAS negative group $(P<0.05)$. In another Japanese study, K-RAS mutations were detected in $8.3 \%$ of 144 patients [32]. The OS rate of NSCLC patients with wild-type K-RAS was better than that of patients whose tumors harboured mutations of $K$ RAS $(P=0.033)$. Miyake et al. analysed tumor tissue from 187 NSCLC patients, among which $8 \%$ harboured a $K$ RAS mutation [33]. In this study, patients with wild-type $K$-RAS had a significantly better survival rate than those with mutant $K$-RAS $(P=0.0369)$. In another study Marks et al. evaluated the prognostic role of EGFR and K-RAS in 296 resected lung adenocarcinomas [34]. Patients were stratified on EGFR and K-RAS mutation, K-RAS mutation or absence of EGFR and K-RAS mutation. In the absence of targeted therapies, 3 -years OS was $90 \%, 76 \%$, and $66 \%$ for patients with $E G F R$ mutations, $E G F R / K$-RAS wild type and $K-R A S$-mutations, respectively. The difference in survival between EGFR-mutated group and K-RAS mutated group was statistically significant $(P=0.009)$. In 2005 a systematic review and meta-analysis of 28 studies including a total of 3620 patients showed that presence of $K-R A S$ mutations confers a significantly worse prognosis, with a combined HR of 1.35 for OS in the random effect model [35]. In a subgroup analysis according to histology, $K-R A S$ mutation resulted in a statistically significant prognostic factor for survival only for adenocarcinoma (HR 1.59). 


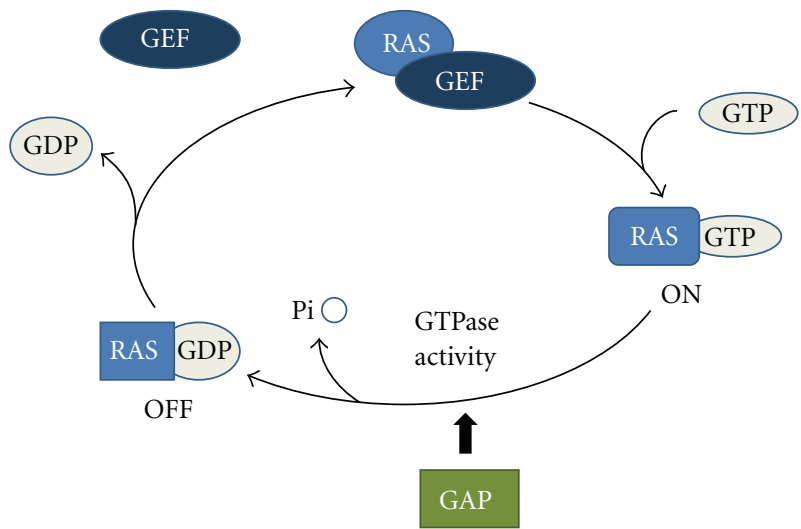

FIGURE 1: Ras activation/deactivation cycle by GEF (guanine exchange factors) and GAP (GTPase activating proteins).

TABLE 1: Prognostic value of $K-R A S$ mutations.

\begin{tabular}{lccc}
\hline Author & Total number of patients & K-RAS mutations (\%) & Survival $(P$-value $)$ \\
\hline Tsao et al. [24] & 450 & 26.0 & 0.4 \\
Schiller et al. [25] & 184 & 24.0 & 0.38 \\
Graziano et al. [26] & 213 & 16.4 & 0.33 \\
Keohavong et al. [27] & 173 & 32 & 0.74 \\
Lu et al. [28] & 94 & 34 & 0.52 \\
Slebos et al. [29] & 69 & 27.5 & $0.002^{*}$ \\
Nelson et al. [30] & 365 & 22.1 & $0.009^{*}$ \\
Fukuyama et al. [31] & 159 & 6.9 & $<0.05^{*}$ \\
Huang et al. [32] & 144 & 8.3 & $0.03^{*}$ \\
Miyake et al. [33] & 187 & 8.0 & 0.037 \\
Marks et al. [34] & 296 & $17 \%$ & NR \\
\hline
\end{tabular}

NR: not reported.* Statistically significant.

Available data suggest that $K$-RAS mutations represent a negative prognostic factor particularly in patients populations with high incidence of EGFR mutations, such as in adenocarcinoma and in Asiatic patients. A possible explanation is that in adenocarcinoma and in Asiatic patients there is a high incidence of EGFR mutations that are considered a positive prognostic factor. In fact, in the study conducted by Marks et al., OS was significantly worse in lung adenocarcinomas with $K$-RAS mutations when compared to patients harbouring EGFR mutations [34].

\section{K-RAS Mutation as Predictive Biomarker}

4.1. Chemotherapy. Recent data suggested that $K-R A S$ mutations may affect the outcome of NSCLC patients receiving chemotherapy (Table 2). In the adjuvant setting, data from the JBR10 trial suggested no benefit from adjuvant chemotherapy in $K$-RAS mutated patients (HR 0.95, $P=$ $0.87)[24,36]$. In the LACE-BIO pooled analysis the prognostic and predictive role of $K-R A S$ mutations was investigated in 1751 patients treated with adjuvant chemotherapy [37]. Among evaluable patients, 304 (19.7\%) harboured K-RAS mutations with no effect on survival (HR 1.18, $P=0.09$ ).
Several studies investigated the influence of $K$-RAS mutations on sensitivity to chemotherapy in advanced NSCLC. Camps et al. analyzed $K-R A S$ status in plasma samples from 308 advanced NSCLC patients treated with cisplatin and docetaxel. No difference in PFS (5.4 versus 5.7 months, $P=$ 0.2 ) or OS (10.0 versus 9.0 months, $P=0.5$ ) was detected between $K$-RAS wild-type and K-RAS mutant patients [38]. Another study retrospectively analyzed 162 chemotherapynaïve patients with locally advanced/metastatic NSCLC who received first-line chemotherapy [39]. Presence of K-RAS mutations did not affect response to chemotherapy (RR, $26.5 \%$ for $K$-RAS wild type versus $25 \%$ for $K$-RAS mutant; $P=0.87$ ) nor time to progression (TTP, 4.2 months for $K$-RAS mutant versus 4.7 months for $K$-RAS wild type; $P=0.42$ ). Furthermore, no significant difference in survival was detected between $K$-RAS wild type and $K$-RAS-mutated patients (14.5 versus 18.5 months for mutations positive and wild-type $K-R A S$ patients, respectively; $P=0.52$ ).

Overall, these data indicate that $K-R A S$ mutations have no role in response prediction to standard chemotherapy in NSCLC and, therefore, such test should not be used in clinical practice. 
TABLE 2: Predictive value of $K-R A S$ mutations on overall survival in patients treated with chemotherapy.

\begin{tabular}{lcccc}
\hline Author & Setting & Total number of patients & K-RAS mutations (\%) & Survival (HR/P value) \\
\hline Tsao et al. [24] & Adjuvant & 450 & 26.0 & $0.95 / 0.87$ \\
Tsao et al. [37] & Adjuvant & 1751 & 19.7 & $1.18 / 0.09$ \\
Camps et al. [38] & Advanced & 308 & 8.8 & NR/0.51 \\
Kalikaki et al. [39] & Advanced & 162 & 22.6 & NR/0.52 \\
\hline
\end{tabular}

HR: hazard ratio; NR: not reported.

4.2. EGFR-TKIs. K-RAS is a critical downstream effector of the EGFR pathway (Figure 2). Therefore, there is a biologic rationale supporting the hypothesis that NSCLC tumors with $K$-RAS mutations are intrinsically resistant to EGFR-directed therapies. In fact, mutations in this gene may produce constitutive activation of the kinase that may overrule the inhibition of EGFR signaling. Initial studies in small cohorts of NSCLC showed lack of response to EGFR-TKIs in patients harboring K-RAS mutations [40-43]. Giaccone et al. analyzed $K-R A S$ status in patients treated with frontline erlotinib and found that none of 10 mutated patients responded to anti-EGFR treatment [40]. Absence of response to erlotinib was reported in another phase II trial in elderly patients. In this study tissue samples from 41 patients were analyzed for $K$-RAS mutations and all the 6 mutated patients identified were refractory to erlotinib [41]. Pao et al. investigated the role of $K-R A S$ mutations in 60 lung adenocarcinomas treated with gefitinib or erlotinib; $K$ RAS mutations were identified in $9(24 \%)$ of 38 patients refractory to either drug, whereas no mutation was detected in 21 sensitive patients [42]. A retrospective analysis of $K$ RAS mutations in patients treated with EGFR-TKIs was conducted by Massarelli et al. In this study $16(22.8 \%)$ of 70 patients had a K-RAS mutation and all of them (100\%) had progressive disease during the treatment [43]. These studies suggested an association between $K-R A S$ mutations and an absence of response to EGFR-TKIs. More recently, two metaanalyses showed that the presence of $K-R A S$ mutations was associated with lack of response to EGFR-TKIs in NSCLC patients $[44,45]$. Nevertheless, both meta-analyses were insufficient to determine the association between $K-R A S$ status and PFS and OS.

Table 3 reports data on $K-R A S$ mutational status and its relationship with survival in phase III trials with anti-EGFR therapy. In the TRIBUTE study, comparing chemotherapy and chemotherapy plus erlotinib, patients with $K-R A S$ mutations had significantly shorter survival when treated with chemotherapy plus erlotinib, suggesting a possible detrimental effect of TKIs in patients harbouring such mutations [46]. The BR.21 trial, evaluating erlotinib versus placebo in second- and third-line setting, showed a survival advantage for erlotinib in the overall population (6.7 versus 4.7 months, HR 0.70; $P<0.001$ ) [13]. Two hundred and six samples were available for $K-R A S$ analysis and in $16 \%$ of cases a $K-R A S$ mutation was detected. In the Cox model, the interaction between $K$-RAS mutation status and treatment suggested a lack of benefit from erlotinib in patients with mutations $(P<0.09)$. Importantly, on multivariate analysis, the presence of $K-R A S$ mutation was not predictive for a differential treatment effect $(P=0.13)$ [47].

A potential benefit in survival produced by erlotinib in $K-R A S$ mutated NSCLC has been reported in the SATURN trial, a large phase III trial randomizing 889 patients who did not progress after first-line chemotherapy, to receive erlotinib or placebo as maintenance treatment $[14,48]$. Four hundred ninety-three $(55.4 \%)$ tumor samples were analyzed for $K$ RAS mutations. Patients treated with erlotinib experienced longer PFS irrespectively of $K-R A S$ mutational status, with a marginal even if not significant survival improvement in the $K$-RAS mutant population (HR 0.79). Another maintenance study, the ATLAS trial, evaluated maintenance treatment with bevacizumab plus placebo or erlotinib in metastatic NSCLC patients not progressing after 4 cycles of platinumbased chemotherapy. The addition of erlotinib significantly reduced the risk of progression (HR 0.72), with the highest benefit observed in the EGFR mutated patients [15]. Analysis of $K-R A S$ mutations highlighted longer PFS for $K-R A S$ wild type patients treated with bevacizumab/erlotinib (HR 0.66, $\log$-rank $P=0.0105)$ but no difference for $K$-RAS-mutant patients (HR 0.92, log-rank $P=0.76$ ) between the two arms. Finally, in the INTEREST study, a large phase III trial comparing gefitinib and docetaxel as second-line therapy in metastatic NSCLC, $18 \%$ of patients harboured K-RAS mutations $[16,49]$. No differences in PFS and response rates were detected in both treatment arms according to $K-R A S$ status, with no evidence of any differential survival effect $(P=0.51)$.

Therefore, although patients harbouring a K-RAS mutation do not respond to EGFR-TKIs, a minimal survival effect cannot be excluded. For such reason, at present, $K-R A S$ testing is not recommended for precluding an EGFR-TKI therapy to any NSCLC patient.

4.3. Anti-EGFR Monoclonal Antibody. A second strategy aimed at inhibiting EGFR signalling is the use of monoclonal antibodies binding the extracellular domain of the receptor. Two large phase III trials investigated the combination of cetuximab, a human-murine chimeric anti-EGFR IgG monoclonal antibody, with chemotherapy versus chemotherapy alone $[50,51]$. In the FLEX trial, 1125 patients with EGFR expressing advanced NSCLC were randomized to receive first-line cisplatin/vinorelbine with or without cetuximab [50]. The addition of cetuximab to chemotherapy led to a significant but clinically marginal survival improvement (11.3 versus 10 months, HR $0.87, P=0.044)$ with an increased risk of toxicity, in particular febrile neutropenia. Similar 


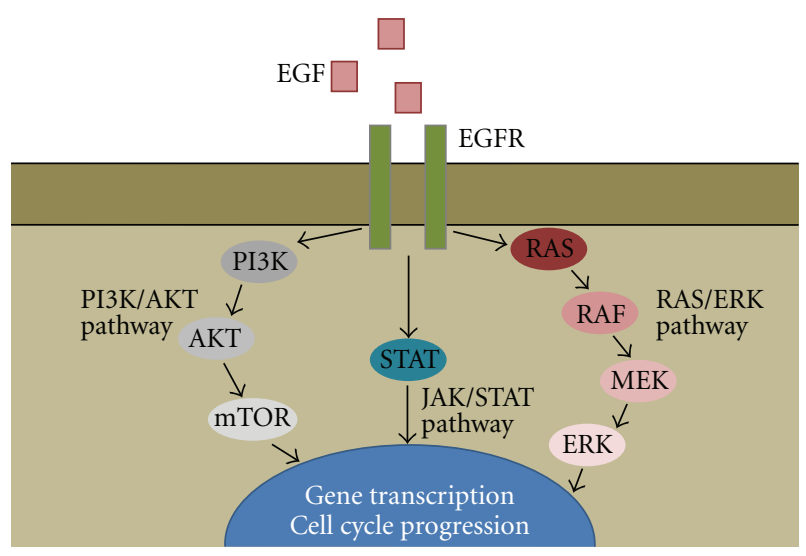

FIGURE 2: EGFR signalling pathway.

TABLE 3: KRAS and sensitivity to anti-EGFR agents in phase III trial.

\begin{tabular}{|c|c|c|c|c|c|}
\hline Trial & $\begin{array}{l}\text { Anti-EGFR } \\
\text { agent }\end{array}$ & $\begin{array}{c}\text { Total number of } \\
\text { patients }(n)\end{array}$ & $\begin{array}{l}\text { Patients tested } \\
\text { for } K R A S(n)\end{array}$ & $\begin{array}{c}\text { KRAS } \\
\text { mutant } n(\%)\end{array}$ & $\begin{array}{c}\text { Survival in KRAS } \\
\text { mutant (HR) }\end{array}$ \\
\hline TRIBUTE [46] & Gefitinib & 1079 & 264 & $55(21)$ & $2.1^{*}$ \\
\hline BR. 21 [47] & Erlotinib & 731 & 206 & $30(15)$ & 1.67 \\
\hline SATURN [48] & Erlotinib & 889 & 493 & $90(18)$ & 0.79 \\
\hline ATLAS [15] & Erlotinib & 768 & NR & NR & 0.92 \\
\hline INTEREST [49] & Gefitinib & 1466 & 275 & $49(18)$ & 0.91 \\
\hline FLEX [57] & Cetuximab & 1125 & 379 & $72(19)$ & 1.0 \\
\hline BMS099 [56] & Cetuximab & 676 & 202 & $35(17)$ & 0.95 \\
\hline
\end{tabular}

HR: hazard ratio; NR: not reported. * Statistically significant.

results were observed in the BMS099 trial, a phase III trial that randomly assigned 676 chemonaïve NSCLC patients to carboplatin plus a taxane versus the same chemotherapy regimen plus cetuximab [51]. Notably, patients were enrolled into the study regardless of EGFR expression. Although a nonsignificant trend toward longer survival (9.6 versus 8.3 months HR $0.89, P=0.17$ ) was reported, the primary end point of improved PFS in the cetuximab arm was not met (4.4 versus 4.2 months, $P=0.2$ ). Based on these studies results, European Medicine Agency (EMA) recently rejected cetuximab approval for advanced NSCLC. This decision clearly highlights the need for biomarkers useful in selecting patients potentially candidate to cetuximab therapy. A recent biomarker analysis of FLEX trial has highlighted a survival benefit in NSCLC patients overexpressing EGFR even in the absence of a PFS benefit [52]. This led to a new submission to EMA in March 2011.

The lack of benefit from anti-EGFR monoclonal antibodies in colorectal cancer patients with $K-R A S$ mutations has been demonstrated [53-55]. The status of $K-R A S$ gene has been investigated even in FLEX and BMS099 trials. In the BMS099 study K-RAS mutant patients treated with cetuximab plus chemotherapy had a trend toward improved PFS and OS than those treated only with chemotherapy [56].
Similarly, in the FLEX trial, $K-R A S$ gene testing failed to identify patients not benefiting from cetuximab and showed similar survival between $K-R A S$ mutant and wild type patients regardless of treatment [57].

These results demonstrate that, unlike colorectal cancer case, the negative predictive value of $K-R A S$ mutations in NSCLC remains unclear. A possible explication of the different role of $K-R A S$ mutation in lung and colorectal cancer has been recently proposed. Danenberg et al. analyzed K-RAS mutation status in 2693 colorectal and lung specimens [58]. Surprisingly, different types of $K-R A S$ mutations were detected in lung and colorectal cancer, with a significant predominance of DNA K-RAS transversions in NSCLC, likely linked to tobacco exposure. The ratio of base transversions to transitions was 3.27 versus 0.77 $(p<001)$ in NSCLC and colorectal cancer, respectively. Tobacco-carcinogenesis-associated $\mathrm{G}>\mathrm{T}$ transversions (codon 12 GGT $>$ TGT plus GGT $>$ GGT) represented $61 \%$ of $K-R A S$ mutations in NSCLC and 39\% in colorectal cancer $(P<0.001)$. It is possible that the distinct mutation pattern and biological function may contribute to differences in predictive value for cetuximab therapy between NSCLC and colorectal cancer. 


\section{Conclusion}

$K-R A S$ mutation testing is a validated biomarker in clinical practice to predict anti-EGFR treatment outcome in colorectal cancer. In a significant fraction of NSCLC, particularly adenocarcinoma and smokers, a K-RAS mutation is detectable, but its prognostic and predictive role remains unclear. Although this event is generally considered associated to a worse prognosis and resistance to several drugs, including EGFR-TKIs, available data are conflicting, not supporting the use of $K-R A S$ testing in clinical practice for selection of NSCLC.

Unfortunately, although $K-R A S$ mutations are one of the most commonly occurring oncogene aberrations in human cancer, no specific treatment is currently available. A new hope for $K-R A S$ mutant patients is represented by novel drugs currently under investigation in phase II and III trials [59]. More recently, scientists uncovered a crack in the molecular armor of RAS, a binding pocket of functional significance that could provide the long-sought attack point for a therapeutic agent [60]. Twenty-five compounds with affinity for binding to RAS oncoproteins were identified by nuclear magnetic resonance spectroscopy. Although all these compounds demonstrated weak affinity for RAS protein and inability to completely knock out the oncoprotein, they represent the first generation of RAS inhibitors, opening a new notable way for research of other compounds able to prevent RAS activation. While waiting for new drugs, the continuous collaboration between basic scientists and clinical researchers is the most relevant way to give hope to our cancer patients.

\section{Abbreviations}

NSCLC: Non-small-cell lung cancer

EGFR: Epidermal Growth Factor Receptor

ALK: Anaplastic Lymphoma Kinase

TKIs: $\quad$ Tyrosine kinase inhibitors

RR: $\quad$ Response rate

PFS: $\quad$ Progression-free survival

OS: Overall survival

GTP: Guanosine triphosphate

MAPK: Mitogen-activated protein kinase

PI3K: Phosphoinositide 3-kinase

STAT: Signal transducer and activator of transcription

TTP: $\quad$ Time to progression

EMA: European Medicine Agency

\section{Acknowledgment}

In part supported by the Italian Association for Cancer Research (AIRC).

\section{References}

[1] A. Jemal, R. Siegel, J. Xu, and E. Ward, "Cancer statistics, 2010," CA Cancer Journal for Clinicians, vol. 60, no. 5, pp. 277$300,2010$.
[2] J. H. Schiller, D. Harrington, C. P. Belani et al., "Comparison of four chemotherapy regimens for advanced non-small-cell lung cancer," New England Journal of Medicine, vol. 346, no. 2, pp. 92-98, 2002.

[3] G. V. Scagliotti, P. Parikh, J. Von Pawel et al., "Phase III study comparing cisplatin plus gemcitabine with cisplatin plus pemetrexed in chemotherapy-naive patients with advancedstage non-small-cell lung cancer," Journal of Clinical Oncology, vol. 26, no. 21, pp. 3543-3551, 2008.

[4] M. Reck, J. Von Pawel, P. Zatloukal et al., "Phase III trial of cisplatin plus gemcitabine with either placebo or bevacizumab as first-line therapy for nonsquamous non-small-cell lung cancer: AVAiL," Journal of Clinical Oncology, vol. 27, no. 8, pp. 1227-1234, 2009.

[5] I. B. Weinstein, "Cancer: addiction to oncogenes - the Achilles heal of cancer," Science, vol. 297, no. 5578, pp. 63-64, 2002.

[6] G. H. Fisher, S. L. Wellen, D. Klimstra et al., "Induction and apoptotic regression of lung adenocarcinomas by regulation of a K-Ras transgene in the presence and absence of tumor suppressor genes," Genes and Development, vol. 15, no. 24, pp. 3249-3262, 2001.

[7] T. S. Mok, Y. L. Wu, S. Thongprasert et al., "Gefitinib or carboplatin-paclitaxel in pulmonary adenocarcinoma," New England Journal of Medicine, vol. 361, no. 10, pp. 947-957, 2009.

[8] J. S. Lee, K. Park, S.-W. Kim et al., "A randomized phase III study of gefitinib (IRESSA) versus standard chemotherapy (gemcitabine plus cisplatin) as a first-line treatment for neversmokers with advanced or metastatic adenocarcinoma of the lung," Journal of Thoracic Oncology, vol. 4, supplement 19, abstract PRS 4, 2009.

[9] T. Mitsudomi, S. Morita, Y. Yatabe et al., "Gefitinib versus cisplatin plus docetaxel in patients with non-small-cell lung cancer harbouring mutations of the epidermal growth factor receptor (WJTOG3405): an open label, randomised phase 3 trial," The Lancet Oncology, vol. 11, no. 2, pp. 121-128, 2010.

[10] M. Maemondo, A. Inoue, K. Kobayashi et al., "Gefitinib or chemotherapy for non-small-cell lung cancer with mutated EGFR," New England Journal of Medicine, vol. 362, no. 25, pp. 2380-2388, 2010.

[11] C. Zhou, Y.-L. Wu, G. Chen et al., "Efficay results from the randomised phase II OPTIMAL (CTNOG 0802) study comparing first-line erlotinib versus carboplatin (CBDCA) plus gemcitabine (GEM), in chinese advanced non-small cell lung cancer (NSCLC) patients (pts) with EGFR activating mutation," Annals of Oncology, vol. 21, supplement 8, abstract LBA 13, 2010.

[12] C.-H. Yang, M. Fukuoka, T. S. Mok et al., "Finall overall survival (OS) results from a phase III, randomised, open-label, first-line study of gefitinib $(\mathrm{G}) \mathrm{v}$ carboplatin/paclitaxel $(\mathrm{C} / \mathrm{P})$ in clinically selected patients with advanced non-small-cell lung cancer (NSCLC) in Asia (IPASS)," Annals of Oncology, vol. 21, supplement 8, abstract LBA 2, 2010.

[13] F. A. Shepherd, J. R. Pereira, T. Ciuleanu et al., "Erlotinib in previously treated non-small-cell lung cancer," New England Journal of Medicine, vol. 353, no. 2, pp. 123-132, 2005.

[14] F. Cappuzzo, T. Ciuleanu, L. Stelmakh et al., "Erlotinib as maintenance treatment in advanced non-small-cell lung cancer: a multicentre, randomised, placebo-controlled phase 3 study," The Lancet Oncology, vol. 11, no. 6, pp. 521-529, 2010.

[15] V. A. Miller, P. O'Connor, C. Soh, and F. Kabbinavar, "A randomized, double-blind, placebo-controlled, phase IIIb trial (ATLAS) comparing bevacizumab (B) therapy with or 
without erlotinib (E) after completion of chemotherapy with $\mathrm{B}$ for first-line treatment of locally advanced, recurrent, or metastatic non-small cell lung cancer (NSCLC)," Journal of Clinical Oncology, vol. 27, supplement 18, abstract LBA8002, 2009.

[16] E. S. Kim, V. Hirsh, T. Mok et al., "Gefitinib versus docetaxel in previously treated non-small-cell lung cancer (INTEREST): a randomised phase III trial," The Lancet, vol. 372, no. 9652, pp. 1809-1818, 2008.

[17] T. Ciuleanu, L. Stelmakh, S. Cicenas et al., "Efficacy and safety of erlotinib versus chemotherapy in second-line advanced non small cell lung cancer (NSCLC) with poor prognosis: the phase III TITAN study," Lung Cancer, vol. 71, no. 2, supplement, pp. S44-S47, 2011.

[18] C. J. Allegra, J. M. Jessup, M. R. Somerfield et al., "American society of clinical oncology provisional clinical opinion: testing for KRAS gene mutations in patients with metastatic colorectal carcinoma to predict response to anti-epidermal growth factor receptor monoclonal antibody therapy," Journal of Clinical Oncology, vol. 27, no. 12, pp. 2091-2096, 2009.

[19] J. L. Bos, "Ras oncogenes in human cancer: a review," Cancer Research, vol. 49, no. 17, pp. 4682-4689, 1989.

[20] S. Rodenhuis, M. L. Van de Wetering, and W. J. Mooi, "Mutational activation of the K-ras oncogene. A possible pathogenetic factor in adenocarcinoma of the lung," New England Journal of Medicine, vol. 317, no. 15, pp. 929-935, 1987.

[21] A. Jemal, R. Siegel, E. Ward et al., "Cancer statistics, 2008," CA Cancer Journal for Clinicians, vol. 58, no. 2, pp. 71-96, 2008.

[22] S. Forbes, J. Clements, E. Dawson et al., “Cosmic 2005," British Journal of Cancer, vol. 94, no. 2, pp. 318-322, 2006.

[23] G. J. Riely, M. G. Kris, J. L. Marks et al., "Frequency and distinctive spectrum of KRAS mutations in never smokers with lung adenocarcinoma," Journal of Clinical Oncology, vol. 26, supplement 15, abstract 8006, 2008.

[24] M. S. Tsao, S. Aviel-Ronen, K. Ding et al., "Prognostic and predictive importance of $\mathrm{p} 53$ and RAS for adjuvant chemotherapy in non-small-cell lung cancer," Journal of Clinical Oncology, vol. 25, no. 33, pp. 5240-5247, 2007.

[25] J. H. Schiller, S. Adak, R. H. Feins et al., "Lack of prognostic significance of $\mathrm{p} 53$ and $\mathrm{K}$-ras mutations in primary resected non-small-cell lung cancer on E4592: a laboratory ancillary study on an eastern cooperative oncology group prospective randomized trial of postoperative adjuvant therapy," Journal of Clinical Oncology, vol. 19, no. 2, pp. 448-457, 2001.

[26] S. L. Graziano, G. P. Gamble, N. B. Newman et al., "Prognostic significance of K-ras codon 12 mutations in patients with resected stage I and II non-small-cell lung cancer," Journal of Clinical Oncology, vol. 17, no. 2, pp. 668-675, 1999.

[27] P. Keohavong, M. A. A. DeMichele, A. C. Melacrinos, R. J. Landreneau, R. J. Weyant, and J. M. Siegfried, "Detection of Kras mutations in lung carcinomas: relationship to prognosis," Clinical Cancer Research, vol. 2, no. 2, pp. 411-418, 1996.

[28] C. Lu, J. C. Soria, X. Tang et al., "Prognostic factors in resected stage I non-small-cell lung cancer: a multivariate analysis of six molecular markers," Journal of Clinical Oncology, vol. 22, no. 22, pp. 4575-4583, 2004.

[29] R. J. C. Slebos, R. E. Kibbelaar, O. Dalesio et al., "K-Ras oncogene activation as a prognostic marker in adenocarcinoma of the lung," New England Journal of Medicine, vol. 323, no. 9, pp. 561-565, 1990.

[30] H. H. Nelson, D. C. Christiani, E. J. Mark, J. K. Wiencke, J. C. Wain, and K. T. Kelsey, "Implications and prognostic value of
K-ras mutation for early-stage lung cancer in women," Journal of the National Cancer Institute, vol. 91, no. 23, pp. 2032-2038, 1999.

[31] Y. Fukuyama, T. Mitsudomi, K. Sugio, T. Ishida, K. Akazawa, and K. Sugimachi, "K-ras and p53 mutations are an independent unfavourable prognostic indicator in patients with nonsmall-cell lung cancer," British Journal of Cancer, vol. 75, no. 8, pp. 1125-1130, 1997.

[32] C. L. Huang, T. Taki, M. Adachi et al., "Mutations of p53 and $\mathrm{K}$-ras genes as prognostic factors for non-small cell lung cancer," International Journal of Oncology, vol. 12, no. 3, pp. 553-563, 1998.

[33] M. Miyake, M. Adachi, C. L. Huang, M. Higashiyama, K. Kodama, and T. Taki, "A novel molecular staging protocol for non-small cell lung cancer," Oncogene, vol. 18, no. 14, pp. 2397-2404, 1999.

[34] J. L. Marks, S. Broderick, Q. Zhou et al., "Prognostic and therapeutic implications of EGFR and KRAS mutations in resected lung adenocarcinoma," Journal of Thoracic Oncology, vol. 3, no. 2, pp. 111-116, 2008.

[35] C. Mascaux, N. Iannino, B. Martin et al., "The role of RAS oncogene in survival of patients with lung cancer: a systematic review of the literature with meta-analysis," British Journal of Cancer, vol. 92, no. 1, pp. 131-139, 2005.

[36] T. Winton, R. Livingston, D. Johnson et al., "Vinorelbine plus cisplatin vs. observation in resected non-small-cell lung cancer," New England Journal of Medicine, vol. 352, no. 25, pp. 2589-2597, 2005.

[37] M. S. Tsao, P. Hainaut, A. Bourredjem et al., "LACE-bio pooled analysis of the prognostic and predictive value of KRAS mutation in completely resected non-small cell lung cancer (NSCLC)," Annals of Oncology, vol. 21, supplement 8, abstract 4218, 2010.

[38] C. Camps, E. Jantus-Lewintre, A. Cabrera et al., "The identification of KRAS mutations at codon 12 in plasma DNA is not a prognostic factor in advanced non-small cell lung cancer patients," Lung Cancer, vol. 72, pp. 365-369, 2011.

[39] A. Kalikaki, A. Koutsopoulos, D. Hatzidaki et al., "Clinical outcome of patients with non-small cell lung cancer receiving front-line chemotherapy according to EGFR and K-RAS mutation status," Lung Cancer, vol. 69, no. 1, pp. 110-115, 2010.

[40] G. Giaccone, M. G. Ruiz, T. Le Chevalier et al., "Erlotinib for frontline treatment of advanced non-small cell lung cancer: a phase II study," Clinical Cancer Research, vol. 12, no. 20, pp. 6049-6055, 2006.

[41] D. M. Jackman, B. Y. Yeap, N. I. Lindeman et al., "Phase II clinical trial of chemotherapy-naïve patients $\geq 70$ years of age treated with erlotinib for advanced non-small-cell lung cancer," Journal of Clinical Oncology, vol. 25, no. 7, pp. 760766, 2007.

[42] W. Pao, T. Y. Wang, G. J. Riely et al., "KRAS mutations and primary resistance of lung adenocarcinomas to gefitinib or erlotinib," PLoS Medicine, vol. 2, no. 1, pp. 0057-0061, 2005.

[43] E. Massarelli, M. Varella-Garcia, X. Tang et al., "KRAS mutation is an important predictor of resistance to therapy with epidermal growth factor receptor tyrosine kinase inhibitors in non-small cell lung cancer," Clinical Cancer Research, vol. 13, no. 10, pp. 2890-2896, 2007.

[44] H. Linardou, I. J. Dahabreh, D. Kanaloupiti et al., "Assessment of somatic k-RAS mutations as a mechanism associated with resistance to EGFR-targeted agents: a systematic review and meta-analysis of studies in advanced non-small-cell lung 
cancer and metastatic colorectal cancer," The Lancet Oncology, vol. 9, no. 10, pp. 962-972, 2008.

[45] C. Mao, L. X. Qiu, R. Y. Liao et al., "KRAS mutations and resistance to EGFR-TKIs treatment in patients with non-small cell lung cancer: a meta-analysis of 22 studies," Lung Cancer, vol. 69 , no. 3, pp. 272-278, 2010.

[46] D. A. Eberhard, B. E. Johnson, L. C. Amler et al., "Mutations in the epidermal growth factor receptor and in KRAS are predictive and prognostic indicators in patients with nonsmall-cell lung cancer treated with chemotherapy alone and in combination with erlotinib," Journal of Clinical Oncology, vol. 23, no. 25, pp. 5900-5909, 2005.

[47] C. Q. Zhu, G. Da Cunha Santos, K. Ding et al., "Role of KRAS and EGFR as biomarkers of response to erlotinib in National Cancer Institute of Canada clinical trials group study BR.21," Journal of Clinical Oncology, vol. 26, no. 26, pp. 4268-4275, 2008.

[48] W. Brugger, N. Triller, M. Blasinska-Morawiec et al., "Prospective molecular marker analyses of EGFR and KRAS from a randomized, placebo-controlled study of erlotinib maintenance therapy in advanced non-small-cell lung cancer," Journal of Clinical Oncology, vol. 29, no. 31, pp. 4113-4120, 2011.

[49] J. Y. Douillard, F. A. Shepherd, V. Hirsh et al., "Molecular predictors of outcome with gefitinib and docetaxel in previously treated non-small-cell lung cancer: data from the randomized phase III INTEREST trial," Journal of Clinical Oncology, vol. 28, no. 5, pp. 744-752, 2010.

[50] R. Pirker, J. R. Pereira, A. Szczesna et al., "Cetuximab plus chemotherapy in patients with advanced non-small-cell lung cancer (FLEX): an open-label randomised phase III trial," The Lancet, vol. 373, no. 9674, pp. 1525-1531, 2009.

[51] T. J. Lynch, T. Patel, L. Dreisbach et al., "Cetuximab and first-line taxane/carboplatin chemotherapy in advanced nonsmall-cell lung cancer: results of the randomized multicenter phase III trial BMS099," Journal of Clinical Oncology, vol. 28, no. 6, pp. 911-917, 2010.

[52] R. Pirker, J. R. Pereira, J. Von Pawel et al., "EGFR expression as a predictor of survival for first-line chemotherapy plus cetuximab in patients with advanced non-small-cell lung cancer: analysis of data from the phase 3 FLEX study," The Lancet Oncology, vol. 13, no. 1, pp. 33-42, 2012.

[53] F. Di Fiore, F. Blanchard, F. Charbonnier et al., "Clinical relevance of KRAS mutation detection in metastatic colorectal cancer treated by Cetuximab plus chemotherapy," British Journal of Cancer, vol. 96, no. 8, pp. 1166-1169, 2007.

[54] S. Khambata-Ford, C. R. Garrett, N. J. Meropol et al., "Expression of epiregulin and amphiregulin and $\mathrm{K}$-ras mutation status predict disease control in metastatic colorectal cancer patients treated with cetuximab," Journal of Clinical Oncology, vol. 25, no. 22, pp. 3230-3237, 2007.

[55] A. Lièvre, J.-B. Bachet, V. Boige et al., "KRAS mutations as an independent prognostic factor in patients with advanced colorectal cancer treated with cetuximab," Journal of Clinical Oncology, vol. 26, no. 3, pp. 374-379, 2008.

[56] S. Khambata-Ford, C. T. Harbison, L. L. Hart et al., "Analysis of potential predictive markers of cetuximab benefit in BMS099, a phase III study of cetuximab and first-line taxane/carboplatin in advanced non-small-cell lung cancer," Journal of Clinical Oncology, vol. 28, no. 6, pp. 918-927, 2010.

[57] K. J. O’Byrne, U. Gatzemeier, I. Bondarenko et al., "Molecular biomarkers in non-small-cell lung cancer: a retrospective analysis of data from the phase 3 FLEX study," The Lancet Oncology, vol. 12, no. 8, pp. 795-805, 2011.
[58] K. D. Danenberg, P. P. Grimminger, P. C. Mack et al., "KRAS mutations (MTs) in non-small cell lung cancer (NSCLC) versus colorectal cancer (CRC): implications for cetuximab therapy," ASCO Meeting Abstracts, vol. 28, no. 15, abstract 10529, 2010.

[59] L. Sequist, W. Akerley, W. Brugger, D. Ferrari, E. Garmey, and D. Gerber, "Final results from ARQ197-209: a global randomized placebo-controlled phase 2 clinical trial of erlotinib plus ARQ197 versus erlotinib plus placebo in previously treated EGFR-inhibitor naive patients with advanced nonsmall cell lung cancer (NSCLC)," Annals of Oncology, vol. 21, supplement 8, abstract viii122, 2010.

[60] T. Maurer, L. Garrenton, A. Oh et al., "Drugging the undraggable: small-molecule inhibition of the Ras oncoprotein," Molecular Biology of the Cell, vol. 22, supplement, Abstract 24, 2011. 

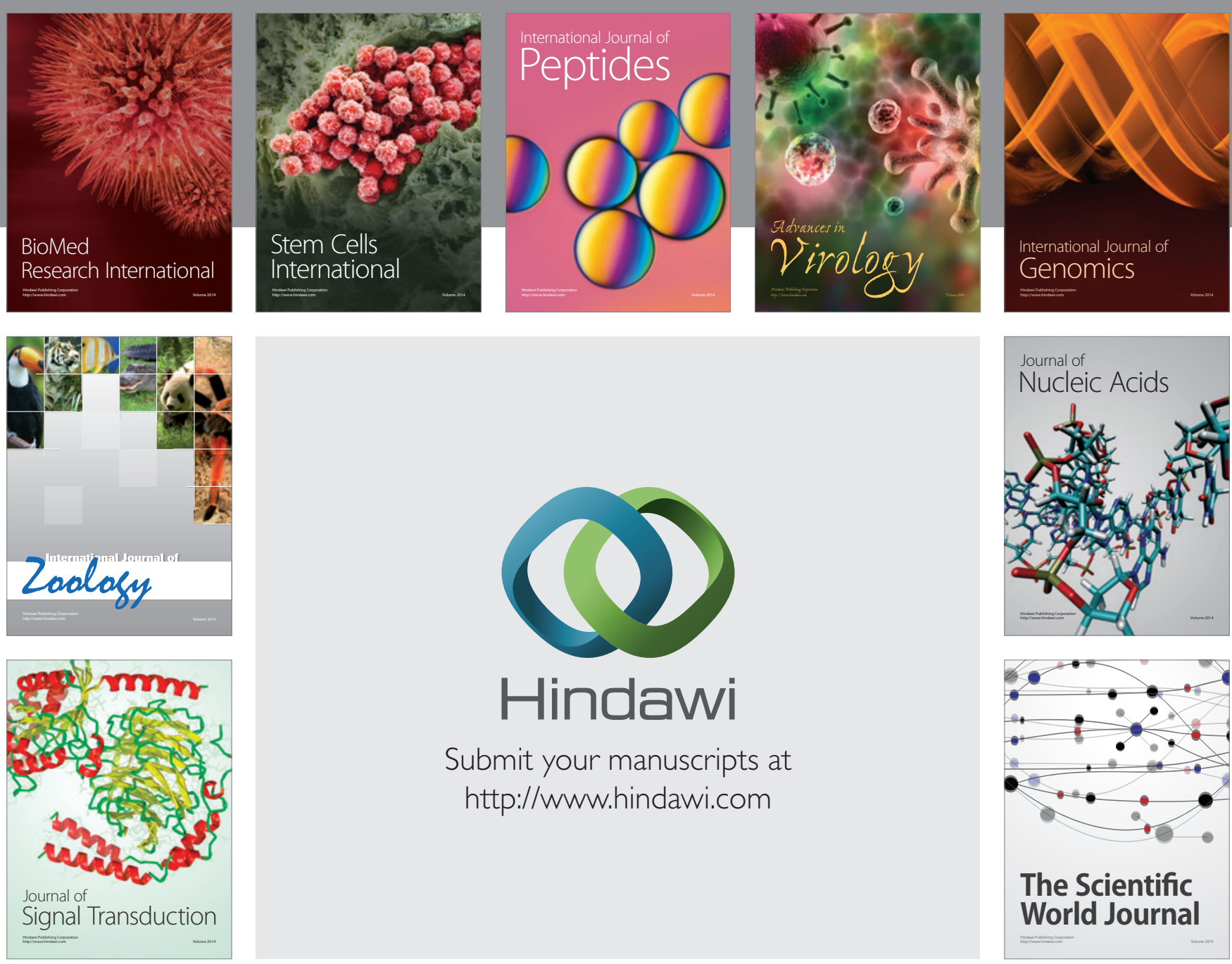

Submit your manuscripts at

http://www.hindawi.com
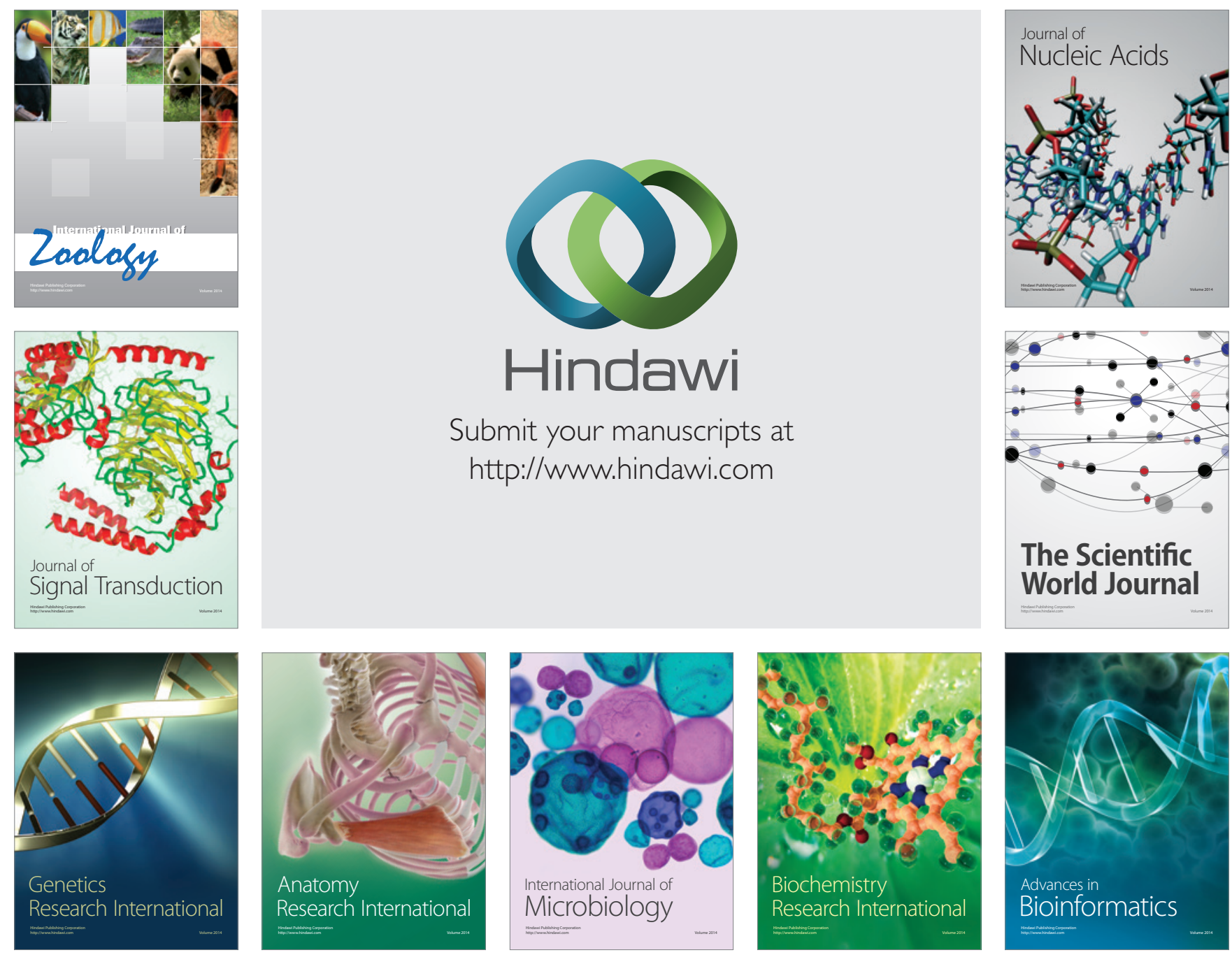

The Scientific World Journal
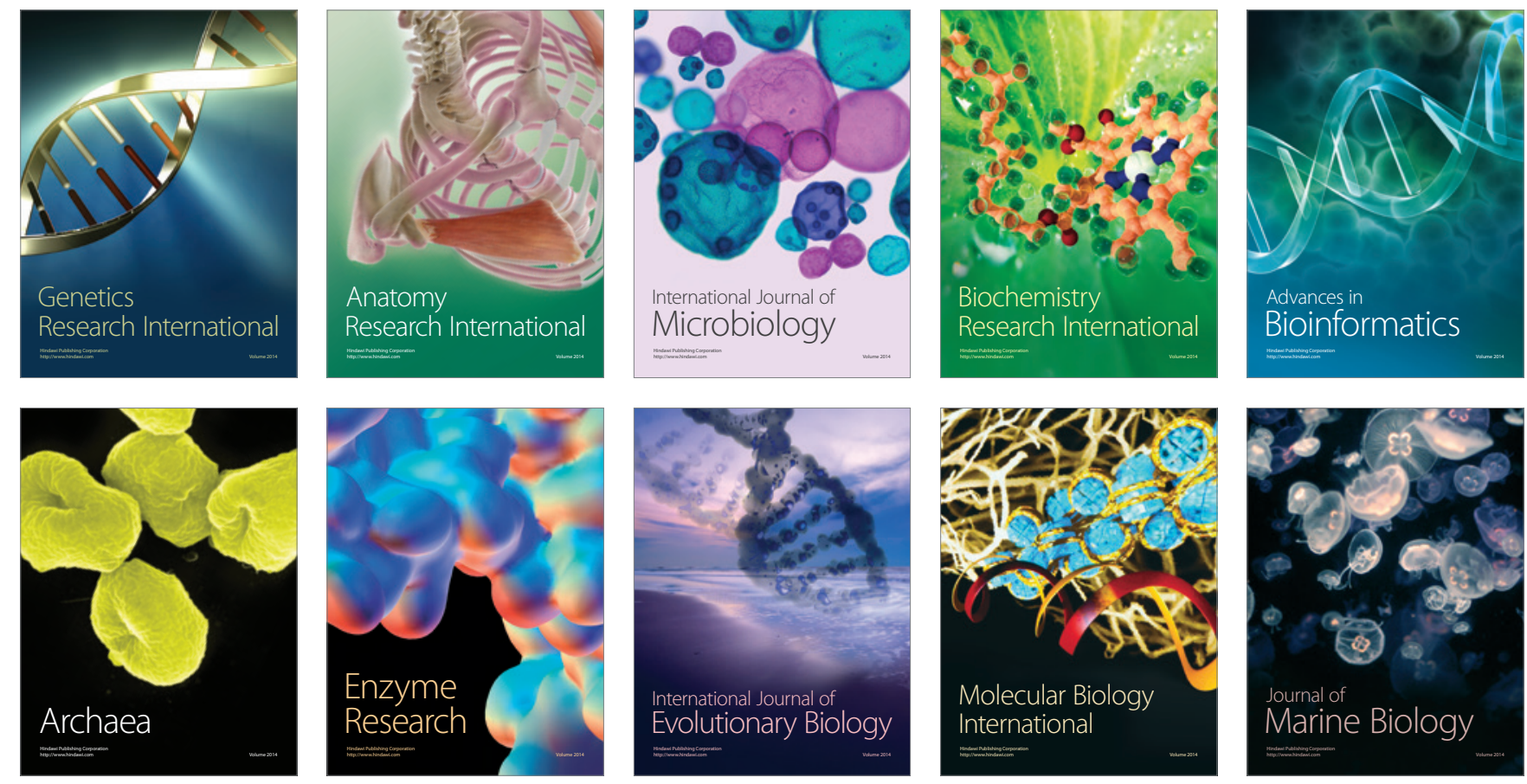